\title{
THE BOHNENBLUST-HILLE INEQUALITY COMBINED WITH AN INEQUALITY OF HELSON
}

\author{
DANIEL CARANDO, ANDREAS DEFANT, AND PABLO SEVILLA-PERIS
}

(Communicated by Alexander Iosevich)

\begin{abstract}
We give a variant of the Bohenblust-Hille inequality which, for certain families of polynomials, leads to constants with polynomial growth in the degree.
\end{abstract}

\section{INTRODUCTION}

Hardy and Littlewood showed in [7] that there exists a constant $K>0$ such that for every $f \in H^{1}$ we have

$$
\left(\int_{\mathbb{D}}|f(z)|^{2} d m(z)\right)^{1 / 2} \leq K \int_{\mathbb{T}}|f(w)| d \sigma(w),
$$

where $d m$ and $d \sigma$ denote respectively the normalised Lebesgue measures on the complex unit disc $\mathbb{D}$ and the torus (or unit circle) $\mathbb{T}$. Equivalently, this means that the Hardy space $H_{1}(\mathbb{T})$ is contained in the Bergman space $B_{2}(\mathbb{D})$. Shapiro [12, pp. 117-118] showed that the inequality holds with $K=\pi$, and Mateljevic [10] (see also [11,13]) showed that actually the constant could be taken to be $K=1$. A simple reformulation of the Bergman norm then gives that if $\sum_{n=0}^{\infty} a_{n} z^{n}$ is the Fourier series expansion of $f \in H^{1}(\mathbb{D})$, we have

$$
\left(\sum_{n=0}^{\infty} \frac{\left|a_{n}\right|^{2}}{n+1}\right)^{1 / 2} \leq \int_{\mathbb{T}}|f(w)| d \sigma(w) .
$$

A few years later Helson in [9] generalised this inequality to functions in $N$ variables. For $n \in \mathbb{N}$ denote by $d(n)$ the number of divisors and by $p^{\alpha}=p_{1}^{\alpha_{1}} \cdots p_{k}^{\alpha_{k}}$ the prime decomposition of $n$. Then we have that for every $f \in H^{1}\left(\mathbb{T}^{N}\right)$ with Fourier series expansion $\sum_{\alpha \in \mathbb{N}_{0}^{N}} c_{\alpha} z^{\alpha}$

$$
\left(\sum_{\alpha \in \mathbb{N}_{0}^{N}} \frac{\left|c_{\alpha}\right|^{2}}{d\left(p^{\alpha}\right)}\right)^{1 / 2} \leq \int_{\mathbb{T}^{N}}|f(w)| d \sigma(w) .
$$

Given a multiindex $\alpha$, we write $\alpha+1=\left(\alpha_{1}+1\right) \cdots\left(\alpha_{k}+1\right)$. Note that, with this notation, we have $d\left(p^{\alpha}\right)=\alpha+1$.

Received by the editors February 7, 2014.

2010 Mathematics Subject Classification. Primary 32A05; Secondary 30C10.

Key words and phrases. Bohnenblust-Hille inequality, Helson inequality, polynomials.

The first author was partially supported by CONICET-PIP 0624, PICT 2011-1456 and UBACyT 20020130100474BA.

The second author was partially supported by MICINN MTM2011-22417.

The third author was supported by MICINN MTM2011-22417 and UPV-SP20120700. 
On the other hand, by the Bohnenblust-Hille inequality [4] as presented in [5] there is a constant $C>0$ such that for every $m$-homogeneous polynomial in $N$ variables $P(z)=\sum_{|\alpha|=m} c_{\alpha} z^{\alpha}$ with $z \in \mathbb{C}^{N}$ we have

$$
\left(\sum_{|\alpha|=m}\left|c_{\alpha}\right|^{\frac{2 m}{m+1}}\right)^{\frac{m+1}{2 m}} \leq C^{m} \sup _{z \in \mathbb{D}^{N}}|P(z)| .
$$

The proof of this inequality given in [5] consists basically of two steps: first to decompose the sum in (1.2) as the product of certain mixed sums and second to bound each one of these sums by a term including $\|P\|$, the supremum of $|P|$ on $\mathbb{D}^{N}$. For this second step usually the following result of Bayart 1 is used: for every $m$-homogeneous polynomial in $N$ variables we have

$$
\left(\sum_{|\alpha|=m}\left|c_{\alpha}\right|^{2}\right)^{1 / 2} \leq 2^{m / 2} \int_{\mathbb{T}^{N}}\left|\sum_{|\alpha|=m} c_{\alpha} w^{\alpha}\right| d \sigma(w) .
$$

Very recently, it was proved in [2, Corollary 5.3] that for every $\varepsilon>0$ there exists $\kappa>0$ such that we can take $\kappa(1+\varepsilon)^{m}$ as the constant in (1.2). Our aim in this note is to get a variant of (1.2) by using (1.1) instead of (1.3). With this variant, we see that for polynomials $P$, each of whose monomials involve a uniformly bounded number of variables, the obtained constants have polynomial growth in $m$.

\section{MAIN RESUlt AND SOME REMARKS}

The following is our main result.

Theorem 2.1. Let $\Lambda \subseteq\left\{\alpha \in \mathbb{N}_{0}^{N}:|\alpha|=m\right\}$ be an indexing set. Then for every family $\left(c_{\alpha}\right)_{\alpha \in \Lambda}$ we have

$$
\left(\sum_{\alpha \in \Lambda}\left(\frac{\left|c_{\alpha}\right|}{\sqrt{\alpha+1}}\right)^{\frac{2 m}{m+1}}\right)^{\frac{m+1}{2 m}} \leq m^{\frac{m-1}{2 m}}\left(1-\frac{1}{m-1}\right)^{m-1} \sup _{z \in \mathbb{D}^{N}}\left|\sum_{\alpha \in \Lambda} c_{\alpha} z^{\alpha}\right| .
$$

We give several remarks before we present the proof.

Remarks 2.2.

(1) It is easy to see that $\sqrt{\alpha+1} \leq \sqrt{2}^{m}$. Hence the preceding inequality includes the hypercontractive version of the Bohnenblust-Hille inequality from (1.2) as a special case.

(2) Thanks to the term $\sqrt{\alpha+1}$, the constants in the previous inequality grow much more slowly than the constants in (1.2). Actually, we have

$$
m^{\frac{m-1}{2 m}}\left(1-\frac{1}{m-1}\right)^{m-1}=\frac{\sqrt{m}}{e}(1+o(m)) .
$$

(3) Let $\operatorname{vars}(\alpha)$ denote the number of different variables involved in the monomial $z^{\alpha}$. In other words, $\operatorname{vars}(\alpha)=\operatorname{card}\left\{j: \alpha_{j} \neq 0\right\}$. Given $M$ we consider the set

$$
\Lambda_{N, M}=\left\{\alpha \in \mathbb{N}_{0}^{N}:|\alpha|=m \text { and } \operatorname{vars}(\alpha) \leq M\right\}
$$

(note that if $M \geq N$, then $\Lambda_{N, M}=\Lambda_{N, N}$ ). An application of Lagrange multipliers gives that for any $\alpha \in \Lambda_{N, M}$ we have for every $N$ and $M$,

$$
\alpha+1=\left(\alpha_{1}+1\right) \cdots\left(\alpha_{k}+1\right) \cdots \leq\left(\frac{m}{M}+1\right)^{M} .
$$


Combining this with Theorem 2.1 we obtain for every $m, N, M$,

$$
\begin{aligned}
\left(\sum_{\alpha \in \Lambda_{N, M}}\left|c_{\alpha}\right|^{\frac{2 m}{m+1}}\right)^{\frac{m+1}{2 m}} & \leq\left(\frac{m}{M}+1\right)^{M / 2}\left(\sum_{\alpha \in \Lambda_{N, M}}\left(\frac{\left|c_{\alpha}\right|}{\sqrt{\alpha+1}}\right)^{\frac{2 m}{m+1}}\right)^{\frac{m+1}{2 m}} \\
& \leq\left(\frac{m}{M}+1\right)^{M / 2} m^{\frac{m-1}{2 m}}\left(1-\frac{1}{m-1}\right)^{m-1} \sup _{z \in \mathbb{D}^{N}}\left|\sum_{\alpha \in \Lambda_{N, M}} c_{\alpha} z^{\alpha}\right|
\end{aligned}
$$

hence

$$
\left(\sum_{\alpha \in \Lambda_{N, M}}\left|c_{\alpha}\right|^{\frac{2 m}{m+1}}\right)^{\frac{m+1}{2 m}} \leq 2^{\frac{M}{2}} m^{\frac{M+1}{2}} \sup _{z \in \mathbb{D}^{N}}\left|\sum_{\alpha \in \Lambda_{N}, M} c_{\alpha} z^{\alpha}\right| .
$$

This means that for polynomials whose monomials have a uniformly bounded number $M$ of different variables, we get a Bohnenblust-Hille type inequality with a constant of polynomial growth in $m$. We remark that the dimension $N$ plays no role in this inequality; the only important point here is the number of different variables in each monomial. As a consequence, an analogue of (2.1) holds for $m$-homogeneous polynomials on $c_{0}$ : let $P: c_{0} \rightarrow \mathbb{C}$ be an $m$-homogeneous polynomial and

$$
\Lambda_{M}=\left\{\alpha \in \mathbb{N}_{0}^{(\mathbb{N})}:|\alpha|=m \text { and } \operatorname{vars}(\alpha) \leq M\right\} .
$$

Then for every $M$ and $m$

$$
\left(\sum_{\alpha \in \Lambda_{M}}\left|c_{\alpha}(P)\right|^{\frac{2 m}{m+1}}\right)^{\frac{m+1}{2 m}} \leq 2^{\frac{M}{2}} m^{\frac{M+1}{2}}\|P\|
$$

where the $c_{\alpha}(P)$ are the coefficients of $P$ and $\|P\|$ is the supremum of $|P|$ on the unit ball of $c_{0}$.

(4) In [6, Theorem 5.3] a very general version of the Bohnenblust-Hille inequality is given, involving operators with values on a Banach lattice. A straightforward combination of the proof of Theorem 2.1 (see the final section) and the arguments presented in [6, Theorem 5.3] easily gives a version of Theorem 2.1 in that setting.

\section{THE PROOF}

Let us fix some notation before we prove our main result. We are going to use the following indexing sets:

$$
\begin{gathered}
\mathcal{M}(m, N)=\left\{\boldsymbol{i}=\left(i_{1}, \ldots, i_{m}\right): 1 \leq i_{j} \leq N, j=1, \ldots, m\right\}, \\
\mathcal{J}(m, N)=\left\{\boldsymbol{i} \in \mathcal{M}(m, N) \in: 1 \leq i_{1} \leq \cdots \leq i_{m} \leq N\right\} .
\end{gathered}
$$

In $\mathcal{M}(m, N)$ we define an equivalence relation by $\boldsymbol{i} \sim \boldsymbol{j}$ if there is a permutation $\sigma$ of $\{1, \ldots, N\}$ such that $j_{k}=i_{\sigma(k)}$ for every $k$. With this, if matrix $\left(a_{i_{1}, \ldots, i_{m}}\right)$ is symmetric, then we have

$$
\sum_{\boldsymbol{i} \in \mathcal{M}(m, N)} a_{\boldsymbol{i}}=\sum_{\boldsymbol{i} \in \mathcal{J}(m, N)} \sum_{\boldsymbol{j} \in[\boldsymbol{i}]} a_{\boldsymbol{j}}=\sum_{\boldsymbol{i} \in \mathcal{J}(m, N)} \operatorname{card}[\boldsymbol{i}] a_{\boldsymbol{i}} .
$$

Also, given $i \in \mathcal{M}(m-1, N)$ and $j \in\{1, \ldots, N\}$, for $1 \leq k \leq m-1$ we define $\left(\boldsymbol{i}_{k} j\right)=\left(i_{1}, \ldots, i_{k-1}, j, i_{k}, \ldots, i_{m-1}\right) \in \mathcal{M}(m, N)$ (that is, we put $j$ in the $k$-th position, shifting the rest to the right). 
There is a one-to-one correspondence between $\mathcal{J}(m, N)$ and $\left\{\alpha \in \mathbb{N}_{0}^{N}:|\alpha|=m\right\}$ defined as follows. For each $i$ we define $\alpha=\left(\alpha_{1}, \ldots, \alpha_{N}\right)$ by $\alpha_{r}=\operatorname{card}\left\{j: i_{j}=r\right\}$ (i.e. $\alpha_{r}$ counts how many times $r$ appears in $\boldsymbol{i}$ ); on the other hand, given $\alpha$ we define $\boldsymbol{i}=\left(1,{ }^{\alpha}{ }^{1}, 1, \ldots, N,{ }_{N},{ }_{N}, N\right) \in \mathcal{J}(m, N)$.

Each $m$-homogeneous polynomial on $N$ variables has a unique symmetric $m$ linear form $L: \mathbb{C}^{N} \times \cdots \times \mathbb{C}^{N} \rightarrow \mathbb{C}$ such that $P(z)=L(z, \ldots, z)$ for every $z$. If $\left(c_{\alpha}\right)$ are the coefficients of the polynomial and $a_{i_{1}, \ldots, i_{m}}=L\left(e_{i_{1}}, \ldots, e_{i_{m}}\right)$ is the matrix of $L$, we have $c_{\alpha}=\operatorname{card}[\boldsymbol{i}] a_{\boldsymbol{i}}$, where $\alpha$ and $\boldsymbol{i}$ are related to each other.

Finally, if $\alpha$ and $\boldsymbol{i}$ are related and $p_{1}<p_{2}<\cdots$ denotes the sequence of prime numbers, we will write $p^{\alpha}=p_{1}^{\alpha_{1}} \cdots p_{N}^{\alpha_{N}}=p_{i_{1}} \cdots p_{i_{m}}=p_{\boldsymbol{i}}$.

Proof of Theorem 2.1. We follow essentially the guidelines of the proof of the Bohnenblust-Hille inequality as presented in [5]. First of all let us assume that $c_{\alpha}=0$ for every $\alpha \notin \Lambda$; then we have

$$
\begin{gathered}
\left(\sum_{\alpha \in \Lambda}\left(\frac{\left|c_{\alpha}\right|}{\sqrt{\alpha+1}}\right)^{\frac{2 m}{m+1}}\right)^{\frac{m+1}{2 m}}=\left(\sum_{\boldsymbol{i} \in \mathcal{J}(m, N)}\left|\operatorname{card}[\boldsymbol{i}] \frac{a_{\boldsymbol{i}}}{\sqrt{d\left(p_{\boldsymbol{i}}\right)}}\right|^{\frac{2 m}{m+1}}\right)^{\frac{m+1}{2 m}} \\
=\left(\sum_{\boldsymbol{i} \in \mathcal{M}(m, N)} \frac{1}{\operatorname{card}[\boldsymbol{i}]}\left|\operatorname{card}[\boldsymbol{i}] \frac{a_{\boldsymbol{i}}}{\sqrt{d\left(p_{\boldsymbol{i}}\right)}}\right|^{\frac{2 m}{m+1}}\right)^{\frac{m+1}{2 m}} \\
=\left(\sum_{\boldsymbol{i} \in \mathcal{M}(m, N)}\left|\operatorname{card}[\boldsymbol{i}]^{1-\frac{m+1}{2 m}} \frac{a_{\boldsymbol{i}}}{\sqrt{d\left(p_{\boldsymbol{i}}\right)}}\right|^{\frac{2 m}{m+1}}\right)^{\frac{m+1}{2 m}} .
\end{gathered}
$$

We now use an inequality due to Blei [3, Lemma 5.3] (see also [5, Lemma 1]): for any family of complex numbers $\left(b_{\boldsymbol{i}}\right)_{\boldsymbol{i} \in \mathcal{M}(m, N)}$ we have

$$
\sum_{\boldsymbol{i} \in \mathcal{M}(m, N)}\left|b_{\boldsymbol{i}}\right|^{\frac{2 m}{m+1}} \leq \prod_{k=1}^{m}\left(\sum_{j=1}^{N}\left(\sum_{\boldsymbol{i} \in \mathcal{M}(m-1, N)}\left|b_{\left(\boldsymbol{i},,_{k} j\right)}\right|^{2}\right)^{1 / 2}\right)^{\frac{2}{m-1}} .
$$

Using this and the fact that $\operatorname{card}\left[\left(\boldsymbol{i},,_{k} j\right)\right] \leq m \operatorname{card}[\boldsymbol{i}]$ we get

$$
\begin{aligned}
\left(\sum_{\alpha \in \Lambda}\left(\frac{\left|c_{\alpha}\right|}{\sqrt{\alpha+1}}\right)^{\frac{2 m}{m+1}}\right)^{\frac{m+1}{2 m}} & \leq \prod_{k=1}^{m}\left(\sum_{j=1}^{N}\left(\sum_{\boldsymbol{i} \in \mathcal{M}(m-1, N)}\left|\operatorname{card}\left[\left(\boldsymbol{i},,_{k} j\right)\right]^{\frac{m-1}{2 m}} \frac{a_{\left(\boldsymbol{i}_{k} j\right)}}{\sqrt{d\left(p_{(\boldsymbol{i}, k j}\right)}}\right|^{2}\right)^{1 / 2}\right)^{\frac{1}{m}} \\
& \leq \prod_{k=1}^{m}\left(\sum_{j=1}^{N}\left(\sum_{\boldsymbol{i} \in \mathcal{M}(m-1, N)}\left|\operatorname{card}[\boldsymbol{i}]^{\frac{m-1}{2 m}} m^{\frac{m-1}{2 m}} \frac{a_{\left(\boldsymbol{i}_{k} j\right)}}{\sqrt{d\left(p_{(\boldsymbol{i}, k} j\right)}}\right|^{2}\right)^{1 / 2}\right)^{\frac{1}{m}} \\
& =m^{\frac{m-1}{2 m}} \prod_{k=1}^{m}\left(\sum_{j=1}^{N}\left(\sum_{\boldsymbol{i} \in \mathcal{M}(m-1, N)} \operatorname{card}[\boldsymbol{i}]\left|\frac{a_{\left(\boldsymbol{i}_{k} j\right)}}{\sqrt{d\left(p_{\left(\boldsymbol{i}_{k} j\right)}\right)}}\right|^{2}\right)^{1 / 2}\right)^{\frac{1}{m}}
\end{aligned}
$$

We now bound each one of the sums in the product. We use the fact that the coefficients $a_{\boldsymbol{j}}$ are symmetric. Also, if $q$ divides $p_{i_{1}} \cdots p_{i_{m}}=p_{\boldsymbol{i}}$, then it also divides $p_{i_{1}} \cdots p_{i_{m}} p_{j}=p_{(\boldsymbol{i}, k j)}$; hence $d\left(p_{\boldsymbol{i}}\right) \leq d\left(p_{(\boldsymbol{i}, k j)}\right)$ for every $\boldsymbol{i}$ and every $j$. This 
altogether gives

$$
\begin{aligned}
& \sum_{j=1}^{N}\left(\sum_{\boldsymbol{i} \in \mathcal{M}(m-1, N)} \operatorname{card}[\boldsymbol{i}]\left|\frac{a_{(\boldsymbol{i}, k j)}}{\sqrt{d\left(p_{(\boldsymbol{i}, k} j\right)}}\right|^{2}\right)^{1 / 2} \\
&=\sum_{j=1}^{N}\left(\sum_{\boldsymbol{i} \in \mathcal{J}(m-1, N)} \operatorname{card}[\boldsymbol{i}]^{2} \frac{\left|a_{\left(\boldsymbol{i}_{k} j\right)}\right|^{2}}{d\left(p_{(\boldsymbol{i}, k j)}\right)}\right)^{1 / 2} \\
& \leq \sum_{j=1}^{N}\left(\sum_{\boldsymbol{i} \in \mathcal{J}(m-1, N)} \frac{\left|\operatorname{card}[\boldsymbol{i}] a_{\left(\boldsymbol{i}_{, k} j\right)}\right|^{2}}{d\left(p_{\boldsymbol{i}}\right)}\right)^{1 / 2} .
\end{aligned}
$$

Let us note that what we have here are the coefficients of an $(m-1)$-homogeneous polynomial in $N$ variables. We now use (1.1) to conclude our argument:

$$
\begin{aligned}
& \sum_{j=1}^{N}\left(\sum_{\boldsymbol{i} \in \mathcal{J}(m-1, N)} \frac{\left|\operatorname{card}[\boldsymbol{i}] a_{\left(\boldsymbol{i}_{k} j\right)}\right|^{2}}{d\left(p_{\boldsymbol{i})}\right)}\right)^{1 / 2} \\
& \leq \sum_{j=1}^{N} \int_{\mathbb{T}^{N}}\left|\sum_{\boldsymbol{i} \in \mathcal{J}(m-1, N)} \operatorname{card}[\boldsymbol{i}] a_{\left(\boldsymbol{i}_{, k} j\right)} w_{i_{1}} \cdots w_{i_{m-1}}\right| d \sigma(w) \\
& \leq \int_{\mathbb{T}^{N}} \sum_{j=1}^{N}\left|\sum_{\boldsymbol{i} \in \mathcal{M}(m-1, N)} a_{\left(\boldsymbol{i},{ }_{k} j\right)} w_{i_{1}} \cdots w_{i_{m-1}}\right| d \sigma(w) \\
& \leq \sup _{z \in \mathbb{D}^{N}} \sum_{j=1}^{N}\left|\sum_{\boldsymbol{i} \in \mathcal{M}(m-1, N)} a_{(i, k j)} z_{i_{1}} \cdots z_{i_{m-1}}\right| \\
& =\sup _{z \in \mathbb{D}^{N}} \sup _{y \in \mathbb{D}^{N}}\left|\sum_{j=1}^{N} \sum_{\boldsymbol{i} \in \mathcal{M}(m-1, N)} a_{\left(\boldsymbol{i},{ }_{k} j\right)} z_{i_{1}} \cdots z_{i_{m-1}} y_{j}\right| \\
& \leq\left(1-\frac{1}{m-1}\right)^{m-1} \sup _{z \in \mathbb{D}^{N}}\left|\sum_{\alpha \in \Lambda} c_{\alpha} z^{\alpha}\right|,
\end{aligned}
$$

where the last inequality follows from a result of Harris [8, Theorem 1] (see also [5. (13)]). This completes the proof.

As we have already mentioned, very recently [2, Corollary 5.3] has shown that for every $\varepsilon>0$ there exists $\kappa>0$ such that (1.2) holds with $\kappa(1+\varepsilon)^{m}$. The main idea for the proof is to replace (3.1) by a similar inequality in which we have mixed sums with $k$ and $m-k$ indices (instead of 1 and $m-1$, as we have here). This allows us to use instead of (1.3) the following inequality:

$$
\left(\sum_{|\alpha|=m}\left|c_{\alpha}\right|^{2}\right)^{1 / 2} \leq c_{p}^{m}\left(\int_{\mathbb{T}^{N}}\left|\sum_{|\alpha|=m} c_{\alpha} w^{\alpha}\right|^{p} d \sigma(w)\right)^{\frac{1}{p}} \quad \text { for } 1 \leq p \leq 2 .
$$

A good control on the constants $c_{p}$ (that tend to 1 as $p$ goes to 2 ) gives an improvement on the constant in (1.2) presented in [2]. In our setting, by dividing by $\alpha+1$, we are using (1.1), which already has constant 1 . Hence this new approach does not improve the constants in our setting. 


\section{ACKNOWLEDGEMENT}

The authors wish to thank the referee for a careful reading and for the suggestions and comments that helped to improve the paper.

\section{REFERENCES}

[1] Frédéric Bayart, Hardy spaces of Dirichlet series and their composition operators, Monatsh. Math. 136 (2002), no. 3, 203-236, DOI 10.1007/s00605-002-0470-7. MR1919645 (2003i:42032)

[2] Frédéric Bayart, Daniel Pellegrino, and Juan B. Seoane-Sepúlveda, The Bohr radius of the $n$-dimensional polydisk is equivalent to $\sqrt{(\log n) / n}$, Adv. Math. 264 (2014), 726-746, DOI 10.1016/j.aim.2014.07.029. MR.3250297

[3] Ron C. Blei, Fractional Cartesian products of sets (English, with French summary), Ann. Inst. Fourier (Grenoble) 29 (1979), no. 2, v, 79-105. MR539694 (81h:43008)

[4] H. F. Bohnenblust and Einar Hille, On the absolute convergence of Dirichlet series, Ann. of Math. (2) 32 (1931), no. 3, 600-622, DOI 10.2307/1968255. MR.1503020

[5] Andreas Defant, Leonhard Frerick, Joaquim Ortega-Cerdà, Myriam Ounaïes, and Kristian Seip, The Bohnenblust-Hille inequality for homogeneous polynomials is hypercontractive, Ann. of Math. (2) 174 (2011), no. 1, 485-497, DOI 10.4007/annals.2011.174.1.13. MR2811605 (2012e:32005)

[6] Andreas Defant, Manuel Maestre, and Ursula Schwarting, Bohr radii of vector valued holomorphic functions, Adv. Math. 231 (2012), no. 5, 2837-2857, DOI 10.1016/j.aim.2012.07.016. MR2970467

[7] G. H. Hardy and J. E. Littlewood, Some properties of fractional integrals. II, Math. Z. 34 (1932), no. 1, 403-439, DOI 10.1007/BF01180596. MR1545260

[8] Lawrence A. Harris, Bounds on the derivatives of holomorphic functions of vectors, Analyse fonctionnelle et applications (Comptes Rendus Colloq. Analyse, Inst. Mat., Univ. Federal Rio de Janeiro, Rio de Janeiro, 1972), Actualités Aci. Indust., No. 1367. Hermann, Paris, 1975, pp. 145-163. MR0477773 (57 \#17283)

[9] Henry Helson, Hankel forms and sums of random variables, Studia Math. 176 (2006), no. 1, 85-92, DOI 10.4064/sm176-1-6. MR2263964 (2007i:43004)

[10] M. Mateljević, The isoperimetric inequality in the Hardy class $H^{1}$, Mat. Vesnik 3(16)(31) (1979), no. 2, 169-178. MR613907 (82i:30052)

[11] Miodrag Mateljević, The isoperimetric inequality and some extremal problems in $H^{1}$, Analytic functions, Kozubnik 1979 (Proc. Seventh Conf., Kozubnik, 1979), Lecture Notes in Math., vol. 798, Springer, Berlin, 1980, pp. 364-369. MR577467(81k:30044)

[12] Joel H. Shapiro, Remarks on F-spaces of analytic functions, Banach spaces of analytic functions (Proc. Pelczynski Conf., Kent State Univ., Kent, Ohio, 1976), Lecture Notes in Math., Vol. 604. Springer, Berlin, 1977, pp. 107-124. MR0487412 (58 \#7050)

[13] Dragan Vukotić, The isoperimetric inequality and a theorem of Hardy and Littlewood, Amer. Math. Monthly 110 (2003), no. 6, 532-536, DOI 10.2307/3647909. MR.1984405

Departamento de Matematica - Pab I, Facultad de Ciencias Exactas y Naturales, Universidad de Buenos Aires, 1428 Buenos Aires, Argentina - And - IMAS - COniCET, ARgEnTINA

E-mail address: dcarando@dm.uba.ar

Institut für Mathematik, Universität Oldenburg, D-26111 Oldenburg, Germany E-mail address: andreas.defant@uni-oldenburg.de

Instituto Universitario de Matemática Pura y Aplicada, Universitat Politècnica de VAlÈnCIA, 46022 VAlEnCia, Spain

E-mail address: psevilla@mat.upv.es 